\title{
Desarrollo de habilidades para el aprendizaje de la geometría de los estudiantes del séptimo ciclo de educación básica, 2019
}

\section{Development of skills for learning the geometry of students in the seventh cycle of basic education, 2019}

\author{
Patricia María Ramos Vera ${ }^{1}$ \\ Rosario Pilar Ramos Vera² \\ Fernando Ramos Vera ${ }^{3}$ \\ Mercedes Josefina Ramos Vera ${ }^{4}$ \\ Luis Alberto Núñez Lira
}

Recibido: 20 de diciembre de 2019. Aceptado: 14 de enero de 2020

\section{RESUMEN}

El estudio tuvo la finalidad de determinar la relación entre las habilidades y el aprendizaje de la geometría en los estudiantes del séptimo ciclo de educación básica; donde la población estuvo conformada por 120 estudiantes. El método empleado en la investigación fue el hipotético deductivo, diseño experimental de clase cuasi experimental, de tipo explicativo; la técnica empleada fue el pre y post prueba y el instrumento una evaluación el cual fue sometido a la validez y confiabilidad respectivamente. Los resultados mostraron que los efectos son significativos en el aprendizaje de la geometría en los estudiantes.

Palabras claves: desarrollo de habilidades; aprendizaje; geometría; historia; conocimiento.

\section{ABSTRACT}

The study aimed to determine the relationship between skills and learning geometry in students in the seventh cycle of basic education; where the population was made up of 120 students. . The method used in the investigation was the hypothetical deductive, of experimental design of quasi-experimental class, of explanatory type; the technique used was the pre and posttest and the instrument an evaluation submitted to the validity and reliability. The results showed that the effects are significant in the learning of geometry in students.

Keywords: skills development; learning; geometry; history; knowledge.

1 Universidad Norbert Wiener. Correo electrónico: patriciadaro@gmail.com, ORCID: https://orcid.org/0000-0002-7591-964X 2 Universidad Norbert Wiener. Correo electrónico: rosarioramosvera@gmail.com, ORCID: https://orcid.org/0000-0002-0712-524X

3 Universidad César Vallejo. Correo electrónico: fer.canteno.2003@gmail.com, ORCID: https://orcid.org/0000-0001-7083-253X

4 Universidad César Vallejo. Correo electrónico: rvmercedesj@gmail.com, ORCID: https://orcid.org/0000-0002-8571-8067

5 Universidad César Vallejo. Correo electrónico: Inunezl@ucv.edu.pe, ORCID: https://orcid.org/0000-0003-3542-9117

(c) 2020 Revista Multi-Ensayos. 


\section{INTRODUCCIÓN}

La calidad de aprendizaje de los estudiantes del nivel secundario refleja un alto índice de fracaso escolar, principalmente en el área de matemática; plasmados en los informes de la prueba del Programa Internacional de Estudiantes (2018) y en la Evaluación Nacional Censal de Estudiantes (2018). Frente a este contexto, se han realizado jornadas de capacitación e innovación con los actores educativos para determinar las causas que generan dichos resultados y así poder plantear alternativas de solución a dicha problemática.

En este sentido, la metodología de enseñanza a través del desarrollo de habilidades es una propuesta que permite mejorar los niveles de logro de los estudiantes. Su aplicación adecuada garantiza el desarrollo de aprendizajes significativos, fortaleciendo de esta manera diversas habilidades, capacidades y competencias en los estudiantes. Desde esta perspectiva, urge modificar la enseñanza con la posibilidad de fortalecer relaciones inclusivas entre docentes y estudiantes (D' Ambrosio, 2001).

Los aprendizajes bajo esta perspectiva socio histórica, ya no se basa en una simple acumulación de contenidos que se asocian a estímulos y respuestas; sino, es aquella que se centra primordialmente en la diversidad de experiencias tanto sociales y culturales de los estudiantes, así como también del mismo medio donde se desarrollen.

Por lo tanto, el presente estudio pretende determinar la relación entre las habilidades y el aprendizaje de la matemática. Así, la formulación del problema es ¿Qué relación existe entre las habilidades y el aprendizaje de la geometría en los estudiantes del séptimo ciclo?

Con esta mirada de mejora, se han revisado varias investigaciones quienes contribuyeron a proponer alternativas para mejorar las habilidades matemáticas y por ende los niveles de desempeño de los estudiantes.

Una de ellas radica en la aplicación de la socioformación en el desempeño de los estudiantes en matemática, lo cual ha permitido superar y mejorar significativamente los resultados de aprendizaje; fortaleciendo las habilidades y competencias matemáticas a través de la solución de situaciones problemáticas de su contexto (Peña, 2018). Asimismo, otros estudios sobre el uso comprensivo de conceptos geométricos bajo el modelo de Van Hiele, mediante el empleo de materiales concretos y actividades lúdicas contextualizadas, evidenciaron mejoras significativas en la comprensión de conceptos geométricos en los estudiantes y mejora en la práctica pedagógica de los docentes (Muñoz, 2018 y Fabres, 2016)

Muchos profesionales de educación consideran que la formación docente es trascendental, porque determina el éxito o fracaso en su desempeño. Al respecto, algunos estudios coincidieron que es necesario formar a los futuros docentes con nuevas propuestas en la curricula, didáctica, metodología, contenidos y evaluación; permitiendo desarrollar la creatividad sin perder de vista la conexión con el entorno sociocultural. Asimismo, los logros más significativos señalan que los docentes formados bajo 
esta propuesta son muy creativos, reflexivos, investigadores, capaces de desarrollar un currículo que responda a la realidad sociocultural de los estudiantes. (Blanco, Fernández y Oliveras, 2017; Gabarrete y Albanece, 2015; Martínez y Oliveras, 2015).

Del mismo modo, Gasco y Gómez, (2016) en sus estudios sobre estrategias de aprendizaje, y el desarrollo de capacidades, demostraron que el buen manejo de estrategias fortalece el razonamiento de los estudiantes al momento de resolver problemas. Además, la aplicación de programas centrado en el contexto de los estudiantes, fortalece las conexiones entre la teoría y práctica, permitiendo obtener resultados significativos (Vega, 2014 y Naresh, 2015) aportando significativamente en la identificación y valorización de las experiencias culturales como también apoya las relaciones sociales en el aula (Fuentes, 2013).

En este sentido, es necesario precisar que una estrategia innovadora; se entiende por aquella que permiten aprender significativamente y autónomamente, desarrollando y fortaleciendo habilidades y competencias en diversos contextos (Monereo, 2002).

De lo anterior, nace la importancia de reconocer el empleo adecuado de estrategias, siendo el docente quien debe planificar y prever una secuencia integrada de procedimientos que permitan lograr los propósitos de aprendizaje. Por todo ello, optar por la aplicación de diversas metodologías y estrategias lleva a constituir una práctica innovadora de variadas posibilidades de enseñanza y aprendizaje, en las que considera los contextos socioculturales de la comunidad donde labore.

Bajo esta perspectiva, el término etno hace referencia a contextos socioculturales (lenguas específicas y jergas, códigos de comportamiento, simbologías, prácticas sociales, sensibilidades); matema al conocimiento y tica, al arte o técnica (artefactos, manifestaciones y producciones). Lo que significa que, en la educación actual, ya no se concibe como una transmisión mecánica y descontextualizada; por el contrario, a partir de esta estrategia se genera un espacio de instrumentos culturales variados, en la cual se puedan desarrollar capacidades críticas a la par con la tecnología, esto quiere decir, que está basada en hacer la matemática algo viva, considerando y proponiendo situaciones reales en un determinado tiempo y espacio. (D’ Ambrosio 2014).

En esta línea, es importante que los estudiantes ingresen al mundo de la matemática a través de sus propias vivencias, descubrimientos y reflexiones; de tal manera que conciban al aprendizaje como experiencia progresiva, divertida, formativa y significativa. En consecuencia, partir de situaciones problemáticas que involucren experiencias reales, permitirá trabajar con motivación, entusiasmo y brindar a su vez la posibilidad de fortalecer las habilidades comunicativas en los estudiantes (Alsina, 2007; Teledahl, 2017).

Ello conlleva a los docentes adoptar una postura sociocultural frente a la educación matemática, el cual tiene como esencia tres perspectivas: La primera surge de la interpretación de la matemática como producto sociocultural; la segunda parte como una tarea con motivaciones e implicaciones de naturaleza social; y la tercera, cuando considera el aula un espacio eminentemente social, en el cual se fortalece la interacción e interrelación entre los agentes educativos (Goñi, 2012). 
Entonces, la esencia de la enseñanza radica en recuperar la presencia de las ideas matemáticas en las actividades humanas, basándose en el principio "en todos los momentos de la historia y en todas las civilizaciones las ideas matemáticas están presentes como formas del hacer y saber" (Goñi, 2006).

Por otro lado, considerar la actividad sociocultural en las prácticas pedagógicas no es el único foco del conocimiento matemático, sino se hace necesario complementar actividades universales en la que los estudiantes tengan que manipular objetos y en ella realizar cortes, medias, localizaciones, diseños, juegos y explicaciones. Ello implica, que se debe concebir procesos educativos formales y no formales que conlleven al logro de aprendizajes significativos como también el desarrollo de habilidades; pues no basta solamente con enseñar matemática, también debemos educar acerca de la matemática, mediante la matemática y con la matemática (Bishop, 1999).

Se ha señalado anteriormente, determinar la relación existente entre las habilidades y el aprendizaje de la geometría. Este se construye desde un nivel elemental hasta un nivel más destacado; permitiendo a los estudiantes tomar decisiones, resolver problemas y comprender el mundo real y objetivo a través de la matemática (Hiele, 1960; Novo, Alsina, Marbán y Berciano, 2017).

Una de las características más resaltantes en los estudiantes de séptimo ciclo de educación básica, es que muestran limitaciones en el desarrollo del pensamiento geométrico, debido a la incomprensión de los campos temáticos como también al tipo de enseñanza recibida por los docentes. Al respecto vale precisar que la geometría como disciplina de la matemática modela el espacio (Camargo 2012); ello implica, que está presente en nuestro entorno real, permitiendo al aprendiz la oportunidad de iniciar y lograr un hermoso viaje hacia las formas superiores de pensamiento complejo. Es decir, que la geometría es una disciplina multifacética contextual, debido a que se encuentra y relaciona estrechamente con otras disciplinas que pertenecen a lo social como natural. En consecuencia, se puede precisar que la matemática representa como mediador entre la disciplina misma con situaciones problemáticas del contexto, y es así que a través de la geometría se logra una representación social y cultural de las mismas en la estructura de los estudiantes (Yuanita, Zulnaidi, \& Zakaria 2018).

Ello nos lleva a entender como el desarrollo de este pensamiento está en función de las capacidades y habilidades humanas, tales como noción del espacio, o también la percepción y hasta la visualización; por ello en su dimensión física, nos conlleva a investigar y profundizar sobre las propiedades de los objetos físicos, como el de sus respectivas representaciones con rigor y su nivel abstracción (Camargo y Acosta, 2012).

Por lo tanto, se debe de concebir con otra mirada a la geometría, es decir, como una actividad netamente humana y no necesariamente como una disciplina solo formal, por tanto, es necesario considerar lo empírico, es decir, desde cómo se percibe, se instruye y visualiza el contexto; como también lo teórico, que contempla habilidades de abstracción, así como los conceptuales que contemplan su rigor. En consecuencia, es necesario considerar esta estrecha relación y vinculación con las actividades y experiencias humanas, lo cual involucra necesariamente lo social, cultural, científico y tecnológico. 
Finalmente, la propuesta permitirá erradicar prácticas algorítmicas, memorísticas y descontextualizadas que imparten los docentes en su práctica pedagógica, incorporando desde la planificación, en la ejecución y evaluación actividades geométricas contextualizadas.

Todo lo expuesto anteriormente, llevó a formular los objetivos de investigación, quienes a su vez orientaran los demás procesos del presente estudio. En tanto, el objetivo general relacionar las habilidades y el aprendizaje de la geometría. Del mismo modo la hipótesis general expresa Las habilidades se relaciones con el aprendizaje de la geometría. En consecuencia, los objetivos e hipótesis específicos se formulan en razón a las dimensiones de la variable dependiente "aprendizaje de la geometría", las cuales constituyen la modelación de objetos, la comunicación, uso de estrategias y la argumentación

\section{DESARROLLO}

\section{Método y metodología}

Para dar respuesta a la pregunta de investigación se utilizó el enfoque mixto, el cual es un proceso que permitió recolectar, analizar y vincular datos tanto cuantitativos y cualitativos. Para el enfoque cuantitativo se utilizó un diseño experimental, de clase cuasi experimental de cuatro grupos de Salomón. Para la recogida de datos se aplicó la prueba pre test en dos grupos (uno experimental y otro de control) y la prueba pos test a los 4 grupos y finalmente, se analizaron los datos mediante el software SPSS. Para el enfoque cualitativo se consideró un diseño descriptivo, que ha permitido dar voz a los involucrados de este estudio a través de sus propias palabras, vivencias y perspectivas; el cual permitió conocer la relación existente entre las habilidades y el aprendizaje de la geometría. La recogida de datos por un lado se realizó a través de la técnica de la observación. Por otro lado, se realizó una entrevista a docentes expertos en el área de matemática y la base teórica necesaria permitiendo realizar la triangulación.

\section{RESULTADOS}

Para el enfoque cuantitativo, los resultados obtenidos descriptivamente destacan que el Grupo Experimental 1, en el pre test, tuvo un nivel de logro del 6,7\% y en el Grupo Control 1 se obtuvo el 3,3\%. Los resultados del pos test nos indican que el Grupo Experimental 1 tuvo el logro de 96,7\% y el Grupo Control 6,7\%. Asimismo, el Grupo Experimental 2 en el pos test tuvo el logro total y el Grupo Control 2 el 3,3 \%. Se puede afirmar que ambos grupos de pos test lograron más del $96 \%$ en el aprendizaje de la geometría; tomando en consideración que el grupo experimental 2 no tuvo la influencia de la prueba pre test. 
Tabla 1: Nivel descriptivo de las habilidades: Pre test y pos test, grupo control y experimental

\begin{tabular}{|c|c|c|c|c|c|c|c|c|c|c|c|c|}
\hline & \multicolumn{2}{|c|}{$\begin{array}{c}\text { Experimental } 1 \\
\text { Habilidades } \\
\text { Pre test } \\
\end{array}$} & \multicolumn{2}{|c|}{$\begin{array}{c}\text { Control } 1 \\
\text { Habilidades } \\
\text { Pre test } \\
\end{array}$} & \multicolumn{2}{|c|}{$\begin{array}{c}\text { Experimental } \\
\text { Habilidades } \\
\text { Post test }\end{array}$} & \multicolumn{2}{|c|}{$\begin{array}{c}\text { Control } 1 \\
\text { Habilidades } \\
\text { Post test }\end{array}$} & \multicolumn{2}{|c|}{$\begin{array}{c}\text { Experimental } 2 \\
\text { Habilidades } \\
\text { Post test } \\
\end{array}$} & \multicolumn{2}{|c|}{$\begin{array}{c}\text { Control } 2 \\
\text { Habilidades } \\
\text { Post test }\end{array}$} \\
\hline & $\mathbf{F}$ & $\%$ & $\mathbf{F}$ & $\%$ & $\mathbf{F}$ & $\%$ & $\mathbf{F}$ & $\%$ & $f$ & $\%$ & $\mathbf{F}$ & $\%$ \\
\hline Logro & 2 & 6,7 & 1 & 3,3 & 29 & 96,7 & 2 & 6,7 & 30 & 100,0 & 1 & 3,3 \\
\hline No logro & 28 & 93,3 & 29 & 97,6 & 1 & 3,3 & 28 & 93,3 & 0 & 0,0 & 29 & 96,7 \\
\hline Total & 30 & 100,0 & 30 & 100,0 & 30 & 100,0 & 30 & 100,0 & 30 & 100,0 & 30 & 100,0 \\
\hline
\end{tabular}

En cuanto a la primera habilidad "modela" se puede observar que el Grupo Experimental 1, en el pre test, tuvo un nivel de logro del 6,7\% y en el Grupo Control 1 obtuvo el 16,7\%. Los resultados del pos test nos indican que el Grupo Experimental 1 tuvo el logro de 63,3\% y el Grupo Control 26,7\%. Asimismo, el Grupo Experimental 2 en el pos test tuvo el logro total del 80\% y el Grupo Control 2 el 13,3 \%. Se puede afirmar que ambos grupos de pos test lograron más del $63 \%$ en la habilidad modela objetos; considerando que el grupo experimental 2 no tuvo la influencia de la prueba pre test.

Tabla 2: Nivel descriptivo de la habilidad modela: Pre test y pos test, grupo control y experimental

\begin{tabular}{|c|c|c|c|c|c|c|c|c|c|c|c|c|}
\hline & \multicolumn{2}{|c|}{$\begin{array}{c}\text { Experimental } 1 \\
\text { Modela } \\
\text { Pre test }\end{array}$} & \multicolumn{2}{|c|}{$\begin{array}{c}\text { Control } 1 \\
\text { Modela } \\
\text { Pre test }\end{array}$} & \multicolumn{2}{|c|}{$\begin{array}{c}\text { Experimental } 1 \\
\text { Modela } \\
\text { Post test }\end{array}$} & \multicolumn{2}{|c|}{$\begin{array}{l}\text { Control } 1 \\
\text { Modela } \\
\text { Post test }\end{array}$} & \multicolumn{2}{|c|}{$\begin{array}{c}\text { Experimental } 2 \\
\text { Modela } \\
\text { Post test }\end{array}$} & \multicolumn{2}{|c|}{$\begin{array}{c}\text { Control } 2 \\
\text { Modela } \\
\text { Post test }\end{array}$} \\
\hline & $f$ & $\%$ & $\mathbf{F}$ & $\%$ & $\mathbf{F}$ & $\%$ & $\mathbf{F}$ & $\%$ & $f$ & $\%$ & $\mathbf{F}$ & $\%$ \\
\hline Logro & 2 & 6,7 & 5 & 16,7 & 19 & 63,3 & 8 & 26,7 & 24 & 80,0 & 4 & 13,3 \\
\hline No logro & 28 & 93,3 & 25 & 83,3 & 11 & 36,7 & 22 & 73,3 & 6 & 20,0 & 26 & 86,7 \\
\hline Total & 30 & 100,0 & 30 & 100,0 & 30 & 100,0 & 30 & 100,0 & 30 & 100,0 & 30 & 100,0 \\
\hline
\end{tabular}

Con respecto a la segunda habilidad "comunica" se puede precisar que el Grupo Experimental 1, en el pre test, tuvo un nivel de logro del 43,3\% y en el Grupo Control 1 obtuvo el 30\%. Los resultados del pos test nos indican que el Grupo Experimental 1 tuvo el logro del $90 \%$ y el Grupo Control el $40 \%$. Asimismo, el Grupo Experimental 2 en el pos test tuvo el logro total del 96,7\% y el Grupo Control 2 el 50\%. Se puede afirmar que ambos grupos de pos test lograron más del $96 \%$ en la habilidad comunica, sabiendo que el grupo experimental 2 no tuvo la influencia de la prueba pre test.

Tabla 3: Nivel descriptivo de la habilidad comunica: Pre test y pos test, grupo control y experimental

\begin{tabular}{|c|c|c|c|c|c|c|c|c|c|c|c|c|}
\hline & \multicolumn{2}{|c|}{$\begin{array}{c}\text { Experimental } 1 \\
\text { Comunica } \\
\text { Pre test } \\
\end{array}$} & \multicolumn{2}{|c|}{$\begin{array}{l}\text { Control } 1 \\
\text { Comunica } \\
\text { Pre test }\end{array}$} & \multicolumn{2}{|c|}{$\begin{array}{c}\text { Experimental } 1 \\
\text { Comunica } \\
\text { Post test } \\
\end{array}$} & \multicolumn{2}{|c|}{$\begin{array}{l}\text { Control } 1 \\
\text { Comunica } \\
\text { Post test }\end{array}$} & \multicolumn{2}{|c|}{$\begin{array}{c}\text { Experimental } 2 \\
\text { Comunica } \\
\text { Post test }\end{array}$} & \multicolumn{2}{|c|}{$\begin{array}{l}\text { Control } 2 \\
\text { Comunica } \\
\text { Post test }\end{array}$} \\
\hline & $f$ & $\%$ & $\mathbf{F}$ & $\%$ & $\mathbf{F}$ & $\%$ & $\mathbf{F}$ & $\%$ & $f$ & $\%$ & $f$ & $\%$ \\
\hline Logro & 13 & 43,3 & 9 & 30 & 27 & 90 & 12 & 40 & 29 & 96,7 & 15 & 50 \\
\hline No logro & 17 & 56,7 & 21 & 70 & 3 & 10 & 18 & 50 & 1 & 3,3 & 15 & 50 \\
\hline Total & 30 & 100,0 & 30 & 100,0 & 30 & 100,0 & 30 & 100,0 & 30 & 100,0 & 30 & 100,0 \\
\hline
\end{tabular}


De la misma manera, en la tercera habilidad "usa de estrategias" se puede observar que el Grupo Experimental 1, en el pre test, tuvo un nivel de logro del 50\% y en el Grupo Control 1 obtuvo el 43,3\%. Los resultados del pos test nos indican que el Grupo Experimental 1 tuvo el logro del 86,7\% y el Grupo Control el 43,3\%. Asimismo, el Grupo Experimental 2 en el pos test tuvo el logro total y el Grupo Control 2 el 50\%. Se puede afirmar que ambos grupos de pos test lograron más del $86 \%$ en la habilidad usa estrategias, tomando en consideración que el grupo experimental 2 no tuvo la influencia de la prueba pre test.

Tabla 4: Nivel descriptivo de la habilidad uso de estrategias: Pre test y pos test, grupo control y experimental

\begin{tabular}{|c|c|c|c|c|c|c|c|c|c|c|c|c|}
\hline & \multicolumn{2}{|c|}{$\begin{array}{c}\text { Experimental } 1 \\
\text { Uso de } \\
\text { estrategias } \\
\text { Pre test }\end{array}$} & \multicolumn{2}{|c|}{$\begin{array}{c}\text { Control } 1 \\
\text { Uso de } \\
\text { estrategias } \\
\text { Pre test } \\
\end{array}$} & \multicolumn{2}{|c|}{$\begin{array}{c}\text { Experimental } 1 \\
\text { Uso de } \\
\text { estrategias } \\
\text { Post test } \\
\end{array}$} & \multicolumn{2}{|c|}{$\begin{array}{l}\text { Control } 1 \\
\text { Uso de } \\
\text { estrategias } \\
\text { Post test } \\
\end{array}$} & \multicolumn{2}{|c|}{$\begin{array}{c}\text { Experimental } 2 \\
\text { Uso de } \\
\text { estrategias } \\
\text { Post test } \\
\end{array}$} & \multicolumn{2}{|c|}{$\begin{array}{l}\text { Control } 2 \\
\text { Uso de } \\
\text { estrategias } \\
\text { Post test } \\
\end{array}$} \\
\hline & $\mathbf{f}$ & $\%$ & $\mathbf{F}$ & $\%$ & $\mathbf{F}$ & $\%$ & $\mathbf{F}$ & $\%$ & $\mathbf{f}$ & $\%$ & $\mathbf{f}$ & $\%$ \\
\hline Logro & 15 & 50 & 13 & 43,3 & 26 & 86,7 & 13 & 43,3 & 30 & 100 & 15 & 50 \\
\hline No logro & 15 & 50 & 17 & 56,7 & 4 & 13,3 & 17 & 56,7 & 0 & 0 & 15 & 50 \\
\hline Total & 30 & 100,0 & 30 & 100,0 & 30 & 100,0 & 30 & 100,0 & 30 & 100,0 & 30 & 100,0 \\
\hline
\end{tabular}

Se puede observar que el Grupo Experimental 1, en el pre test, tuvo un nivel de logro del $30 \%$ y en el Grupo Control 1 obtuvo el 36,7\%. Los resultados del pos test nos indican que el Grupo Experimental 1 tuvo el logro del 73,3\% y el Grupo Control el 33,3\%. Asimismo, el Grupo Experimental 2 en el pos test tuvo el logro del $80 \%$ y el Grupo Control 2 el 23,3\%. Se puede afirmar que ambos grupos de pos test lograron más del $73 \%$ en la habilidad argumenta, considerando que el grupo experimental 2 no tuvo la influencia de la prueba pre test.

Tabla 5: Nivel descriptivo de la habilidad argumenta pre test y pos test, grupo control y grupo experimental

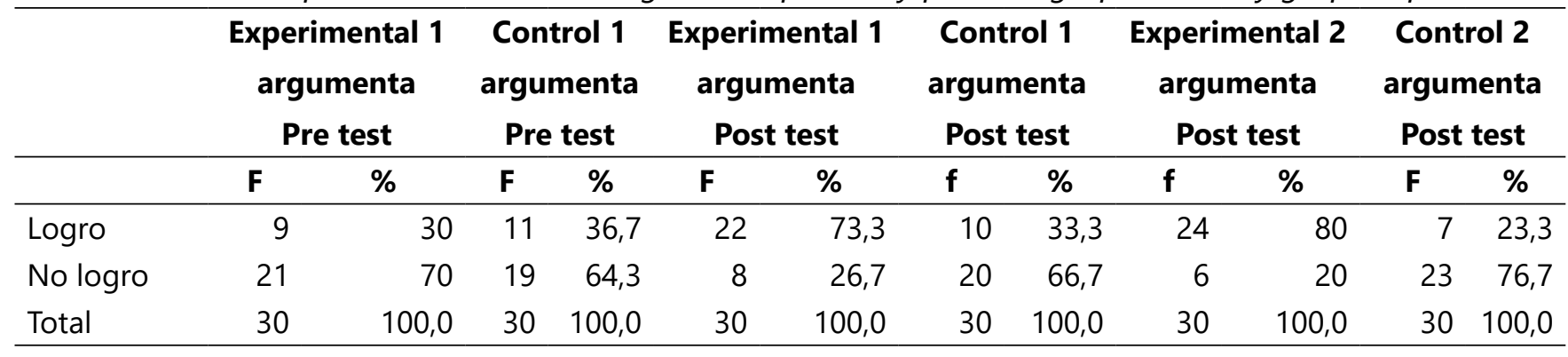

Por otro lado, en cuanto a los resultados obtenidos en la prueba de la hipótesis, se puede deducir que en la posevaluación de las habilidades en los grupos control y experimental, el mayor rango promedio fue el del grupo posexperimental 2 con 97,45; en la posevaluación de la habilidad modela de los grupos control y experimental, el mayor rango promedio fue el del grupo posexperimental 2 con 83,00; en la posevaluación de la habilidad comunica de los grupos control y experimental, el mayor rango promedio fue el del grupo posexperimental 2 con 86,03; en la posevaluación de la habilidad uso de estrategias de los grupos control y experimental, el mayor rango promedio fue el del grupo posexperimental 2 con 
83,30; en la posevaluación de la habilidad argumenta de los grupos control y experimental, el mayor rango promedio fue el del grupo posexperimental 2 con 78,30.

Con respecto a los estadísticos para la prueba de las hipótesis, la significatividad estadística o la prueba de p-valor, nos indican que los grupos son diferentes, en todos los casos, por lo tanto, las ventajas obtenidas, reflejadas en los datos nos indican que las habilidades han generado aprendizajes significativos en el área de matemática -general y específicos de los estudiantes.

Con respecto a los resultados obtenidos en el enfoque cualitativo, al momento de triangular lo vertido por los estudiantes, expertos y teóricos, se puede precisar lo siguiente: La relación entre las habilidades y la habilidad modela objetos con formas geométricas y realiza transformaciones son significativas, ya que la aplicación de diversos materiales educativos contextualizados como láminas con tejidos, juegos, fichas, entre otros; han resultado de sumo interés y motivación para los estudiantes, pues la manipulación de los mismos contribuyó a la construcción significativa de conocimientos geométricos.

Del mismo modo, resultó para la habilidad comunica sobre las formas y relaciones geométricas, pues los estudiantes se sintieron muy motivados e interesados cuando desarrollaron diversas actividades lúdicas como el juego "domino inca", el cual permitió identificar adecuadamente las características de los polígonos y comunicar las propiedades mediante un lenguaje adecuado. Otra situación muy similar, resultó con la habilidad usa estrategias y procedimientos para orientarse en el espacio, pues la participación activa de los estudiantes en el desarrollo de actividades contextualizadas contribuyó a desarrollar los problemas de manera autónoma, para ello seleccionaron, adaptaron y combinaron diversas técnicas, procedimientos y estrategias. Finalmente, en la el desarrollo de la habilidad argumenta relaciones geométricas, los estudiantes mostraron una actitud crítica y creativa, socializando los problemas del contexto con fundamento.

\section{CONCLUSIÓN}

A partir de los hallazgos encontrados se acepta la primera hipótesis específica, la cual establece que los efectos de la etnomatemática son significativos en la capacidad modela objetos con formas geométricas y sus respectivas transformaciones. Estos resultados guardan relación con lo sostenido por Muñoz (2018) y Fabres (2016), quienes obtuvieron no solo la mejora significativa con respecto a la comprensión de conceptos geométricos en los estudiantes; sino también, en la práctica pedagógica docente. Expresan además, la importancia del empleo de materiales concretos y de la planificación de actividades lúdicas contextualizadas, tal como los resultados obtenidos en el presente estudio.

Con respecto a los hallazgos encontrados se acepta la segunda hipótesis específica, la cual establece que los efectos de la etnomatemática son significativos en la capacidad comunicativa de las formas y relaciones geométricas. Estos resultados tienen relación con lo propuesto por Gasco (2017); Romero, Gamarra y Miranda (2017), quienes señalan la influencia de los micro proyectos curriculares etnomatemáticos en el aprendizaje de los estudiantes; pues, la resolución de problemas moviliza y fortalece la capacidad comunicativa en la medida que los estudiantes trabajen colaborativa y cooperativamente. 
Del mismo modo, los hallazgos encontrados permiten aceptar la tercera hipótesis específica, la cual precisa sobre los efectos significativos de la etnomatemática en la capacidad usa estrategias y procedimientos al momento de trazar, medir o estimar distancias y superficies; cuando construyen formas geométricas y realizan transformaciones bi y tridimensionalmente. Al respecto Gasco y Gómez (2016) sostienen que la reconstrucción de prácticas culturales en el aula fortalece el desarrollo del pensamiento lógico en los estudiantes, permitiéndoles emplear diversos procedimientos en la elaboración, planificación, regulación y autorreflexión de procedimientos. En tanto, los autores expresan la importancia de fomentar un clima adecuado que contribuya al desarrollo de un aprendizaje autónomo y creativo.

Los hallazgos encontrados aceptan la cuarta hipótesis específica, que establece que los efectos de la etnomatemática son significativos en la capacidad argumentativa de los estudiantes cuando establecen relaciones geométricas y aplican propiedades. Estas actividades fortalecen directamente esta capacidad cuando justifican, validan ejemplos o contraejemplos, pues ponen en juego el razonamiento inductivo o deductivo. Del mismo modo Vega (2014) y Naresh (2015) afirman que la aplicación de actividades en base a la cultura y el contexto fortalecen las conexiones significativas entre la etnomatemática, la teoría y práctica; ello conlleva a que los estudiantes sean capaces de demostrar soluciones de situaciones problemáticas mediante la argumentación y justificación de sus procedimientos; por lo tanto, precisan la importancia de brindar a los estudiantes la oportunidad de emplear el razonamiento lógico en la solución de situaciones reales.

Finalmente, frente a los hallazgos encontrados anteriormente permite aceptar la hipótesis general, la cual establece que los efectos de la etnomatemática son significativos en el aprendizaje de la geometría. Del mismo modo, los resultados obtenidos por Blanco, Fernández y Oliveras (2017); Gabarrete y Albanece (2015); Martínez y Oliveras (2015) guardan relación con la presente investigación, pues señalan la necesidad de aplicar la etnomatemática como herramienta para la formación docente; para replantear la curricula, didáctica, metodología, contenidos y evaluación, permitiendo mejorar el desempeño de los estudiantes en el aprendizaje de la matemática. Así mismo, Peña (2018) señala el fortalecimiento de las competencias matemáticas en los estudiantes cuando resuelven situaciones problemáticas reales del contexto; Como también aporta significativamente en la identificación cultural y mejora las relaciones sociales en el aula Fuentes (2013)

\section{REFERENCIAS}

Alsina, Á. (2007). El aprendizaje reflexivo en la formación permanente del profesorado: un análisis desde la didáctica de la matemática. Educación Matemática, 2007, vol. 19, núm. 1, p. 99-126. URL: https:// dugi-doc.udg.edu/bitstream/handle/10256/9367/AprendizajeReflexivoPermanente.pdf?sequen.

Blanco, H.; Fernández, A., \& Oliveras, M. (2017). Mathematics Teacher Training from Ethnomathematics: $A$ developmental state. Bolema: Boletim de Educação Matemática, 31(58), 564-589. ISSN 1980-4415.

Bishop, A. (1999). Enculturación matemática. La educación matemática desde una perspectiva cultural. Paidós, México. URL: https://mmsrcapital.files.wordpress.com/2015/03/1991-enculturacic3b3nmatemc3a1tica-alan-j-bishop1.pdf 
Camargo, L., \& Acosta, M. (2012). La geometría, su enseñanza y su aprendizaje. Tecné, Episteme y Didaxis: TED, (32), 4-8. ISSN 0121-3814. URL: http://www.scielo.org.co/scielo.php?script=sci_ arttext\&pid=S0121-38142012000200001.

D'Ambrosio, U. (2014). Las bases conceptuales del Programa Etnomatemática. Revista Latinoamericana de Etnomatemática, 7(2), 100-107. URL: https://www.revista.etnomatematica.org/index.php/ RevLatEm/article/view/126

D'Ambrosio, U. (2001) Etnomatemática: Elo entre las tradições e a modernidad. Colección: Tendencias en educación matemática. Belo Horizonte: Autêtica.

Evaluación Censal de Estuantes ECE (2018). URL: http://umc.minedu.gob.pe/resultados-ece-2018/

Fuentes, C. (2014). Etnomatemática, escuela y aprendizaje de las matemáticas: el caso de la comunidad de Guacamayas, Tesis de Maestría. Universidad Distrital Francisco José De Caldas. Facultad de Ciencias y Educación. Boyacá, Colombia. URL: http://www.etnomatematica.org/publica/trabajos_ maestria/Tesis11_Fuentes_Ago2014.pdf

Fabres, R. (2016). Estrategias metodológicas para la enseñanza y el aprendizaje de la geometría, utilizadas por docentes de segundo ciclo, con la finalidad de generar una propuesta metodológica atingente a los contenidos. Estudios pedagógicos (Valdivia), 42(1), 87-105. ISSN 0718-0705. DOI: http://dx.doi. org/10.4067/S0718-07052016000100006

Fuentes, C. (2013). Etnomatemática y escuela: algunos lineamientos para su integración. Revista Científica, ISSN 0124-2253. DOI: https://doi.org/10.14483/23448350.5483.

Gavarrete, E., \& Albanese, V. (2015). Etnomatemáticas de signos culturales y su incidencia en la formación de maestros. Revista Latinoamericana de Etnomatemática: Perspectivas Socioculturales de la Educación Matemática, 8(2), 299-315. URL: http://funes.uniandes.edu.co/6837/1/ Albanese2015Etnomatematicas.pdf

Gasco T. (2017). Diferencias en el uso de estrategias en el aprendizaje de las matemáticas en enseñanza secundaria según el sexo. Cuadernos de Investigación Educativa, 8(1), 47-59. ISSN 1510-2432

Gasco T. (2016). El empleo de estrategias en el aprendizaje de las Matemáticas en Enseñanza Secundaria Obligatoria. Revista de Investigación Educativa, 34(2), 487-502. ISSN 2340-924X. DOI: http://dx.doi. org/10.6018/rie.34.2.222901. URL: https://revistas.um.es/rie/article/view/222901/195101

Goñi, J. M. (2012). Didáctica de las matemáticas. Educatio Siglo XXI, 30(1), 350-352.I SBN 9788499800448. Martínez, O., \& Oliveras, M. L. (2015). Surcando caminos de interculturalidad sustentados en la Etnomatemática. Revista Latinoamericana de Etnomatemática: Perspectivas Socioculturales de la Educación Matemática, 8(2), 341-363. URL: http://funes.uniandes.edu.co/6842/1/ Oliveras2015Surcando.pdf

Monereo, C., Castelló, M., Clariana, M., Palma, M., \& Pérez, M. L. (2000). Estrategias de enseñanza y aprendizaje. Graó. ISBN 970-18-1041-4 DL: 13-45.987-98

Muñoz, A.(2018). Enseñanza y aplicación de conceptos geométricos (Distancia, altura, área y perímetro) a través de actividades agrícolas con el cultivo de fríjol (Phaseolus vulgaris) en los terrenos de la Institución Educativa Rural Obispo, Supía Caldas (Doctoral dissertation, Universidad Nacional de Colombia-Sede Manizales). URL: http://www.bdigital.unal.edu.co/65058/1/1060648621.2018.pdf

Naresh, N. (2015). The role of a critical ethnomathematics curriculum in transforming and empowering learners. Revista Latinoamericana de Etnomatemática: Perspectivas Socioculturales de la Educación Matemática, 8(2), 450-471. URL: http://funes.uniandes.edu.co/6832/1/Naresh2015Role.pdf 
Novo, M. L., Alsina, Á., Marbán, J. M., \& Berciano, A. (2017). Connective Intelligence for Childhood Mathematics Education. Comunicar: Media Education Research Journal, 25(52), 29-39.doi: DOI: https://doi.org/10.3916/C52-2017-03 URL: https://files.eric.ed.gov/fulltext/EJ1169189.pdf

PISA (2015), Como oportunidad de aprendizaje. Buenos Aires: Ministerio de Educación de Argentina, Dirección Nacional de Información y Evaluación de la Calidad Educativa (DINIECE). URL: http:/ / www.oei.es/pisa2009Lectura.pdf 203

Peña, M. (2018). UVE socioformativa: Estrategia didáctica para evaluar la pertinencia de la solución a problemas de contexto. IE Revista de Investigación Educativa de la REDIECH, 9(16), 133-153.

Teledahl, A. (2017). How young students communicate their mathematical problem solving in writing. International journal of mathematical education in science and technology, 48(4), 555-572. URL: https://www.tandfonline.com/doi/abs/10.1080/0020739X.2016.1256447

Vega, R. (2014). Influencia de la etnomatemática en el desarrollo de las capacidades del área de matemática en alumnos del 2do año de secundaria de la I.E. N²0265 Los Atavillos. URL: http://repositorio.ucv. edu.pe/handle/UCV/15097

Yuanita, P., Zulnaidi, H., \& Zakaria, E. (2018). The effectiveness of Realistic Mathematics Education approach: The role of mathematical representation as mediator between mathematical belief and problem solving. Plos one, 13(9), e0204847.URL: https://journals.plos.org/plosone/article/file?id=10.1371/ journal.pone.0204847\&type $=$ printable. 